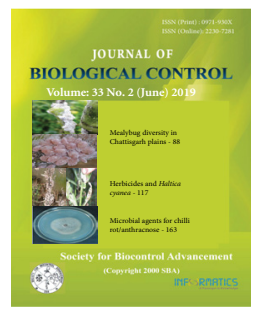

\title{
Evaluation of entomopathogenic fungi against sucking pests of Bhut Jalakia
}

\author{
RUDRA N. BORKAKATI ${ }^{1 *}$, D. K. SAIKIA ${ }^{1}$ and B. RAMANUJAM ${ }^{2}$ \\ ${ }^{1}$ Department of Entomology, Assam Agricultural University, Jorhat - 785013, Assam, India \\ ${ }^{2}$ ICAR-National Bureau of Agricultural Insect Resources, H. A. Farm Post, Bellary Road, Hebbal, Bengaluru - 560024, \\ Karnataka, India \\ *Corresponding author E-mail: rnbk.agri@gmail.com
}

\begin{abstract}
Experiment was conducted to evaluate the efficacy of six different strains of entomopathogenic fungi and one insecticide molecule against sucking pests of Bhut Jalakia during 2014-15 to 2016-17. Bhut Jalakia is one of the hottest chillis in the world, grown extensively in Assam, Nagaland and Manipur. Aphis gossypii Glover, Scirtothrips dorsalis Hood and Bemisia tabaci (Genn.) are the major sucking pests of Bhut Jalakia inflicting damage right from planting to fruiting stages of the crop. Three rounds of biopesticides @ $1 \times 10^{8}$ spores/gm and insecticide (imidacloprid 17.8 SL @ 20 gm a.i./ha) imposed at 3 weeks interval against the pests revealed that imidacloprid 17.8 SL could significantly reduce the mean population of A. gossypii (4.64); S. dorsalis (2.03) and B. tabaci (0.28), closely followed by Beauveria bassiana (NBAIR-Bb-5a) with 7.20, 3.07 and 0.64 per 10 leaves and both the treatments were on par in their efficacy after third spray. The rest of the entomopathogenic fungi were more or less effective in reducing the sucking pests and statistically at par with each other compared to untreated control plot. Highest yield of $52.64 \mathrm{q} /$ ha recorded in imidacloprid treated plot followed by NBAIR strain of Bb-5a with $45.85 \mathrm{q} / \mathrm{ha}$ and had no any significant difference from each other. Minimum yield of $26.78 \mathrm{q} /$ ha was obtained in untreated control plot.
\end{abstract}

KEY WORDS: Bhut Jalakia, biopesticides, entomopathogenic fungi, sucking pests

(Article chronicle: Received: 02-09-2018; Revised: 18-02-2019; Accepted: 25-02-2019)

\section{INTRODUCTION}

Bhut Jalakia, Capsicum chinense Jacq., is one of the hottest chillis in the world, and at the same time has a pleasant and palatable aroma. Bhut Jalakia is a inter specific hybrid between Capsicum chinense and C. frutescens. It is believed to have originated in North-east India. The heat value of Bhut Jalakia is 1,001,304 SHU, which is due to presence of a volatile phenolic amine Capsaicin and dihydrocapsaicin (Sanatombi and Sharma, 2008). In 2007, Guinness Records certified the Bhut Jalakia as the world's hottest chilli pepper, which was 400 times hotter than Tabasco sauce (Baruah et al., 2014). The Assamese word 'Bhut' refers to the typical, large pod size, with high hotness in the fruits (Bhagowati and Changkija, 2009).

The key reasons of drastic reduction of yield of chilli are due to heavy infestations of different types of sucking pests. At vegetative and flowering stage, the crop is the most vulnerable to sucking pests. However, Bhut Jalakia also attacked by almost same kinds of sucking pests of chilli, viz., aphids, Myzus persicae Sulzer, Aphis gossypii Glover; mites, Polyphagotarsonemus latus Banks; thrips, Scirtothrips dorsalis Hood, and jassid, Amrasca biguttula biguttula Ishida (Berke and Sheih, 2000; Begam et. al., 2016; Buragohain et. al., 2017). Indiscriminate and unplanned application of the pesticides by the growers has led to the development of many undesirable problems which become a threat to chilliecosystem causing resurgence of pest, environmental problems, residues and destruction of natural enemy fauna, etc. The residue of ethion and chlorpyrifos was recorded in chillies even after one month of the first application to manage the sucking pests during the vegetative growth of the crop (Mahalingappa et al., 2006). Besides health hazard and environmental pollution, hot quality of Bhut Jalakia is also gradually decreased due to the heavy use of synthetic chemicals including fertiliser and pesticides. Hence, this has promoted research to develop an alternative management strategy which would be safer, effective and economical. Moreover, biological control is now becoming very popular in insect pests control owing to its ecofriendly nature. Entomopathogenic fungi may play a pivotal role to manage the insect pests as safe alternatives to synthetic insecticides. Keeping all these points in view, an effort was made to study the efficacy of certain entomofungal 
pathogens for management of sucking pests of Bhut Jalakia.

\section{MATERIALS AND METHODS}

The Experiment was laid out at Randomised Block Design (RBD) in the Experimental Farm, Department of Horticulture, Assam Agricultural University, Jorhat during 2014-15, 2015-16 and 2016-17. Bhut Jalakia variety "Raja" (local) was raised in $10.5 \mathrm{~m}^{2}$ plot containing 8 treatments including control and was replicated 4 times. To evaluate the effect of entomopathogenic fungi, viz., Metarhizium anisopliae (AAU-Biometa; MRC-0314), M. anisopliae (NBAIRMa-4; NCBI Acc. JF837157 and NBAIRMa-35, NCBI Acc. JQ518481), Beauveria bassiana (Biosona; MTCC- 25040) and B. bassiana (NBAIRBb-5a; NCBI Acc. JF837134 and NBAIRBb-23; NCBI Acc. JF837082) against sucking pests of Bhut Jalakia were used. Moreover, for comparison with chemical, imidacloprid17.8 SL@20 gm a.i./ha was selected in addition to an untreated control check. control check. The entomopathogenic fungi belonged to the culture collection of Assam Agricultural University, Jorhat and National Bureau of Agricultural Insect Resources, Bengaluru. The powder based formulations of these fungi were prepared by Talc formulation was prepared by inoculating one loopful of culture in autoclaved potato dextrose broth (PDB) and incubated for 8 days at $26^{\circ} \mathrm{C}, 150 \mathrm{RPM}$ in incubator shaker. The culture was mixed in 1:1 ratio (sterilized Talc: culture) and dried for 2-3days. Moisture was checked for $8 \%$ and packed for further use. The talc formulation contains 1 x 108 $\mathrm{cfu} / \mathrm{gram}$ where dose is recommended as 5 grams /liter (Ali Derakshan, 2008).

All the plants were subjected to three rounds of foliar sprays of insecticide and bio-pesticide and first were started in 0 (Zero) days of planting and subsequent sprays were imposed at 3 weeks intervals in morning hour without contaminating the adjacent plots. The untreated plots were sprayed with water only.

The population of aphids (Aphis gossypii), thrips (Scirtothripsdorsalis), and white fly (Bemisia tabaci) was recorded from five randomly selected plants considering ten leaves from upper, middle and bottom, one day before and $3^{\text {rd }}$, $7^{\text {th }}$ and $10^{\text {th }}$ day after spraying. Picking of mature fruits was collected at 7-day interval and weight of the healthy fruits was recorded.

\section{RESULTS AND DISCUSSION}

Impact of various entomopathogenic fungi $\left(1 \times 10^{8}\right.$ spores/gm @ 5g/l) along with imidacloprid 17.8 SL @ 20 g. a.i./ha $(0.4 \mathrm{ml} / \mathrm{l})$ taken as standard check against sucking pests of Bhut Jalakia is presented in table 1, 2 and 3. Perusal of data revealed that the population of different sucking pests was at par during pre-spraying in different treatments. Among the various treatments evaluated against Bhut Jalakia, imidacloprid was the most effective and proved to be the best in reducing the population of aphid with $6.25,4.67$ and 3.00 during 2014-15, 2015-16 and 2016-2017, respectively, after third spray. However, it was closely followed by NBAIRBb-5a with 8.50 aphids per 10 leaves and showed no any significant difference between them in their efficacies after $3^{\text {rd }}$ spray during 2014-15, during 2015-16 and 2016-17, NBAIRBb-5a was also, the next best treatment with 8.00 and 5.10 aphid per 10 leaves when compared with imidacloprid $17.8 \mathrm{SL}$. The rest of the entomopathogenic fungi were found to be more or less equally effective in reducing the aphid population of Bhut Jalakia compared to untreated control plot where the aphid population per 10 leaves were $32.00,24.00$ and 15.85 during all the crop seasons (Table 1). The effectiveness of imidacloprid17.8 SL @ 20 g. a.i./ha (0.4 ml/l) against aphids of chilli had also been observed by Patil et al. (2002).

The same results in reducing Aphis gossypii by Verticillium lecanii and Beauveria bassiana was also reported by (Loueiro and Moino, 2006; Kim et al., 2013; Jandricic et al., 2014; Lee et al., 2015; Eidy et al., 2016). Highest mortality of chilli aphid was also achieved due to application of imidacloprid 17.8SL after three days of insecticide spray (Das, 2013).

The same trend of results was also obtained by imidacloprid 17.8SL in suppressing Scirtothrips dorsalis and Bemisia tabaci compared to different entomopathogenic fungi tested in the experiments during 2014-15 to 2016-17. The lowest number of S. dorsalis was recorded 1.25, 2.72 and 2.13 per 10 leaves during the different crop seasons in the imidacloprid 17.8SL treated plot. Similarly, the population of B. tabaci in imidacloprid $17.8 \mathrm{SL}$ treated plot was $0.78,0.76$ and 0.80 per 10 leaves after third spray. Amongst the different entomopathogenic fungi, the next best treatment was NBAIR strain $\mathrm{Bb}-5 \mathrm{a}$, where the $S$. dorsalis and B. tabaci population per 10 leaves were $2.50,3.58,3.13$ and $0.67,0.63,0.64$, respectively, during different crop seasons. As regards to other entomopathogenic fungi, all the biopesticides showed a significant difference in reducing the population of $S$. dorsalis and B. tabaci over the untreated control plot, where the maximum population of $S$. dorsalis $(15.50,12.50$ and 13.60) and B. tabaci $(2.25,2.30$ and 2.27) per 10 leaves was recorded during 2014-15 to 2016-17 (Table 2 and 3).

The present findings were in conformity with Patil et al. (2002) who reported that imidacloprid 17.8 SL was highly effective against sucking pest complex in chilli. Similarly, Jagdish and Purnima (2011) reported that application of 
BORKAKATI et al.

Table 1. Effect of entomopathogenic fungi against Aphis gossypi

\begin{tabular}{|c|c|c|c|c|c|c|c|c|c|c|c|c|c|c|c|}
\hline \multirow[t]{3}{*}{ Treatments } & \multicolumn{4}{|c|}{ 2014-15 } & \multicolumn{4}{|c|}{$2015-16$} & \multicolumn{4}{|c|}{ 2016-17 } & \multirow{2}{*}{\multicolumn{3}{|c|}{$\begin{array}{c}\text { Pooled Mean } \\
\text { Post treatment count }\end{array}$}} \\
\hline & \multirow{2}{*}{$\begin{array}{l}\text { Pre } \\
\text { treat- } \\
\text { ment } \\
\text { count }\end{array}$} & \multicolumn{3}{|c|}{ Post treatment count $*$} & \multirow{2}{*}{$\begin{array}{l}\text { Pre } \\
\text { treat- } \\
\text { ment } \\
\text { count }\end{array}$} & \multicolumn{3}{|c|}{ Post treatment count $*$} & \multirow{2}{*}{$\begin{array}{l}\text { Pre } \\
\text { treat- } \\
\text { ment } \\
\text { count }\end{array}$} & \multicolumn{3}{|c|}{ Post treatment count $*$} & & & \\
\hline & & Ist spray & $\begin{array}{l}\text { II }^{\text {nd }} \\
\text { spray }\end{array}$ & $\begin{array}{l}\text { III }^{\text {rd }} \\
\text { spray }\end{array}$ & & I $^{\text {st }}$ spray & $\begin{array}{l}\text { II }^{\text {nd }} \\
\text { spray }\end{array}$ & $\begin{array}{l}\mathrm{III}^{\mathrm{rd}} \\
\text { spray }\end{array}$ & & I $^{\text {st }}$ spray & $\begin{array}{l}\text { II }^{\text {nd }} \\
\text { spray }\end{array}$ & $\begin{array}{l}\text { III }{ }^{\text {rd }} \\
\text { spray }\end{array}$ & $\begin{array}{l}\text { Ist } \\
\text { spray }\end{array}$ & $\begin{array}{l}\text { II }^{\text {nd }} \\
\text { spray }\end{array}$ & $\begin{array}{l}\mathrm{III}^{\mathrm{rd}} \\
\text { spray }\end{array}$ \\
\hline $\begin{array}{l}\text { Metarhizium anisopliae } \\
\text { (AAU-Biometa) }\end{array}$ & 25.00 & $20.25^{\mathrm{b}}$ & $15.25^{b}$ & $12.00^{\mathrm{b}}$ & 21.56 & $14.83^{\mathrm{bc}}$ & $12.25^{\mathrm{cd}}$ & $10.75^{\mathrm{bc}}$ & 14.55 & $11.45^{\mathrm{b}}$ & $8.00^{\mathrm{bc}}$ & $7.00^{\mathrm{b}}$ & 15.51 & 11.83 & 9.92 \\
\hline $\begin{array}{l}\text { B. bassiana } \\
\text { (AAU-Biosona) }\end{array}$ & 22.00 & $18.00^{\mathrm{bc}}$ & $14.75^{\mathrm{bc}}$ & $11.00^{\mathrm{bc}}$ & 19.75 & $13.67^{\mathrm{c}}$ & $12.25^{\mathrm{cd}}$ & $10.00^{\mathrm{c}}$ & 15.10 & $11.85^{b}$ & $8.30^{\mathrm{b}}$ & $7.05^{b}$ & 14.51 & 11.77 & 9.35 \\
\hline $\begin{array}{l}\text { M. anisopliae } \\
\text { (NBAIRMa-4) }\end{array}$ & 21.00 & $16.75^{\mathrm{cd}}$ & $14.50^{\mathrm{bcd}}$ & $11.25^{\mathrm{bcd}}$ & 19.72 & $14.17^{\mathrm{c}}$ & $13.67^{b}$ & $9.91^{\mathrm{c}}$ & 14.95 & $11.00^{\mathrm{bc}}$ & $7.45^{\mathrm{c}}$ & $7.00^{\mathrm{b}}$ & 13.97 & 11.87 & 9.39 \\
\hline $\begin{array}{l}\text { M. anisopliae } \\
\text { (NBAIRMa-4) }\end{array}$ & 20.50 & $16.75^{\mathrm{cd}}$ & $12.75^{\mathrm{cd}}$ & $10.25^{\mathrm{bc}}$ & 22.59 & $16.17^{b}$ & $15.42^{b}$ & $11.08^{b}$ & 14.30 & $10.95^{\mathrm{bc}}$ & $7.55^{c}$ & $7.05^{b}$ & 14.62 & 11.91 & 9.46 \\
\hline $\begin{array}{l}\text { Beauveria bassiana } \\
\text { (NBAIRBb-5a) }\end{array}$ & 21.00 & $15.75^{\mathrm{cd}}$ & $12.00^{\mathrm{e}}$ & $8.50^{\mathrm{de}}$ & 21.42 & $11.50^{\mathrm{d}}$ & $11.17^{\mathrm{d}}$ & $8.00^{\mathrm{d}}$ & 14.00 & $7.95^{\mathrm{d}}$ & $6.45^{d}$ & $5.10^{\mathrm{c}}$ & 11.73 & 9.87 & 7.20 \\
\hline $\begin{array}{l}\text { B. bassiana } \\
\text { (NBAIBb-23) }\end{array}$ & 21.5 & $17.75^{\mathrm{bc}}$ & $12.50^{\mathrm{de}}$ & $9.00^{\mathrm{d}}$ & 20.17 & $11.33^{\mathrm{d}}$ & $9.84^{\mathrm{e}}$ & $8.75^{\mathrm{d}}$ & 14.65 & $10.25^{\mathrm{c}}$ & $7.55^{\mathrm{c}}$ & $7.30^{\mathrm{b}}$ & 13.11 & 9.96 & 8.35 \\
\hline Imidacloprid 17.8 SL & 21.75 & $14.50^{\mathrm{d}}$ & $9.00^{\mathrm{f}}$ & $6.25^{\mathrm{e}}$ & 20.34 & $8.33^{\mathrm{e}}$ & $6.33^{\mathrm{f}}$ & $4.67^{\mathrm{e}}$ & 15.68 & $5.30^{\mathrm{e}}$ & $4.15^{\mathrm{e}}$ & $3.00^{\mathrm{d}}$ & 9.38 & 6.49 & 4.64 \\
\hline Untreated control & 23.75 & $27.75^{\mathrm{a}}$ & $27.00^{\mathrm{a}}$ & $32.00^{\mathrm{a}}$ & 18.92 & $21.00^{\mathrm{a}}$ & $22.00^{\mathrm{a}}$ & $24.00^{\mathrm{a}}$ & 14.25 & $14.80^{\mathrm{a}}$ & $\begin{array}{c}16.40 \\
a\end{array}$ & $\begin{array}{c}15.85 \\
\mathrm{a}\end{array}$ & 21.18 & 21.80 & 23.95 \\
\hline $\mathrm{CD}=0.05$ & NS & 2.60 & 2.03 & 2.25 & NS & 1.99 & 1.21 & 1.11 & NS & 1.01 & 0.70 & 0.70 & 2.58 & 2.52 & 4.07 \\
\hline $\mathrm{CV} \%$ & & 9.61 & 9.37 & 12.21 & & 9.75 & 6.41 & 6.92 & & 6.58 & 5.76 & 6.42 & 10.32 & 12.05 & 22.59 \\
\hline
\end{tabular}

Means followed by the same letter in a column are not significantly different

*Count/10 leaves

\section{Table 2. Effect of entomopathogenic fungi against Scirtothrips dorsalis}

\begin{tabular}{|c|c|c|c|c|c|c|c|c|c|c|c|c|c|c|c|}
\hline \multirow[t]{3}{*}{ Treatments } & \multicolumn{4}{|c|}{ 2014-15 } & \multicolumn{4}{|c|}{$2015-16$} & \multicolumn{4}{|c|}{$2016-17$} & \multirow{2}{*}{\multicolumn{3}{|c|}{$\begin{array}{c}\text { Pooled Mean } \\
\text { Post treatment count }\end{array}$}} \\
\hline & \multirow{2}{*}{$\begin{array}{l}\text { Pre } \\
\text { treat- } \\
\text { ment } \\
\text { count }\end{array}$} & \multicolumn{3}{|c|}{ Post treatment count $*$} & \multirow{2}{*}{$\begin{array}{l}\text { Pre } \\
\text { treat- } \\
\text { ment } \\
\text { count }\end{array}$} & \multicolumn{3}{|c|}{ Post treatment count $*$} & \multirow{2}{*}{$\begin{array}{l}\text { Pre } \\
\text { treat- } \\
\text { ment } \\
\text { count }\end{array}$} & \multicolumn{3}{|c|}{ Post treatment count $*$} & & & \\
\hline & & $\mathrm{I}^{\mathrm{st}}$ spray & $\begin{array}{l}\text { II }^{\text {nd }} \\
\text { spray }\end{array}$ & III $^{\text {rd }}$ spray & & I $^{\text {st }}$ spray & $\begin{array}{l}\mathrm{II}^{\text {nd }} \\
\text { spray }\end{array}$ & $\begin{array}{l}\mathrm{III}^{\mathrm{rd}} \\
\text { spray }\end{array}$ & & $\mathrm{I}^{\mathrm{st}}$ spray & $\begin{array}{l}\text { II }^{\text {nd }} \\
\text { spray }\end{array}$ & $\begin{array}{l}\text { III }^{\text {rd }} \\
\text { spray }\end{array}$ & $\begin{array}{l}\text { It }^{\text {st }} \\
\text { spray }\end{array}$ & $\begin{array}{l}\mathrm{II}^{\text {nd }} \\
\text { spray }\end{array}$ & $\begin{array}{l}\mathrm{III}^{\mathrm{rd}} \\
\text { spray }\end{array}$ \\
\hline $\begin{array}{l}\text { Metarhizium anisopliae } \\
\text { (AAU-Biometa) }\end{array}$ & 10.25 & $7.00^{\mathrm{b}}$ & $6.00^{\mathrm{b}}$ & $4.50^{\mathrm{b}}$ & 11.92 & $8.33^{\mathrm{b}}$ & $8.75^{\mathrm{b}}$ & $7.00^{\mathrm{b}}$ & 11.10 & $7.65^{\mathrm{bc}}$ & $7.75^{\mathrm{b}}$ & $7.60^{\mathrm{b}}$ & 7.66 & 7.50 & 6.37 \\
\hline $\begin{array}{l}\text { Beauveria bassiana } \\
\text { (AAU-Biosona) }\end{array}$ & 11.00 & $6.50^{\mathrm{bc}}$ & $5.25^{\mathrm{bc}}$ & $3.50^{\mathrm{bc}}$ & 10.58 & $7.00^{\mathrm{c}}$ & $7.33^{\mathrm{c}}$ & $5.83^{\mathrm{c}}$ & 11.90 & $7.80^{\mathrm{b}}$ & $7.75^{\mathrm{b}}$ & $6.40^{\mathrm{c}}$ & 7.10 & 6.78 & 5.24 \\
\hline $\begin{array}{l}\text { M. anisopliae } \\
\text { (NBAIRMa-4) }\end{array}$ & 10.50 & $5.25^{\mathrm{cd}}$ & $4.75^{\mathrm{cd}}$ & $3.50^{\mathrm{bc}}$ & 13.0 & $8.83^{\mathrm{b}}$ & $6.33^{\text {cde }}$ & $5.33 \mathrm{~d}^{\mathrm{c}}$ & 11.65 & $6.78^{\mathrm{c}}$ & $6.45^{\mathrm{c}}$ & $6.08^{\mathrm{c}}$ & 6.95 & 5.84 & 4.97 \\
\hline $\begin{array}{l}\text { M. anisopliae } \\
\text { (NBAIRMa-35) }\end{array}$ & 12.25 & $6.50^{\mathrm{bc}}$ & $5.00^{\mathrm{c}}$ & $3.25^{\mathrm{cd}}$ & 12.92 & $9.83^{\mathrm{b}}$ & $6.42^{\mathrm{cd}}$ & $4.75^{\mathrm{de}}$ & 12.55 & $8.10^{\mathrm{b}}$ & $6.75^{\mathrm{c}}$ & $5.23^{\mathrm{d}}$ & 8.14 & 6.06 & 4.41 \\
\hline $\begin{array}{l}\text { B. bassiana } \\
\text { (NBAIRBb-5a) }\end{array}$ & 9.50 & $3.50^{\mathrm{ef}}$ & $3.00^{\mathrm{de}}$ & $2.50^{\mathrm{cd}}$ & 13.75 & $7.08^{\mathrm{c}}$ & $5.58 \mathrm{~d}^{\mathrm{e}}$ & $3.58^{\mathrm{gf}}$ & 12.70 & $6.65^{\mathrm{c}}$ & $4.45^{\mathrm{d}}$ & $3.13^{\mathrm{e}}$ & 5.74 & 4.34 & 3.07 \\
\hline $\begin{array}{l}\text { B. bassiana } \\
\text { (NBAIRBb-23) }\end{array}$ & 10.75 & $4.25^{\mathrm{de}}$ & $3.25^{\mathrm{d}}$ & $3.00^{\mathrm{cd}}$ & 11.08 & $5.42^{\mathrm{d}}$ & $4.75^{\mathrm{e}}$ & $4.50^{\text {ef }}$ & 10.65 & $6.75^{\mathrm{c}}$ & $5.40^{\mathrm{d}}$ & $5.15^{\mathrm{d}}$ & 5.47 & 4.47 & 4.22 \\
\hline Imidacloprid $17.8 \mathrm{SL}$ & 12.25 & $2.75^{\mathrm{f}}$ & $2.00^{\mathrm{e}}$ & $1.25^{\mathrm{d}}$ & 12.25 & $4.67^{\mathrm{d}}$ & $3.33^{\mathrm{f}}$ & $2.72^{\mathrm{g}}$ & 11.65 & $4.43^{\mathrm{d}}$ & $3.20^{\mathrm{e}}$ & $2.13^{\mathrm{f}}$ & 3.95 & 2.84 & 2.03 \\
\hline Untreated control & 12.25 & $15.75^{\mathrm{a}}$ & $17.25^{\mathrm{a}}$ & $15.50^{\mathrm{a}}$ & 10.67 & $11.75^{\mathrm{a}}$ & $13.17^{\mathrm{a}}$ & $12.50^{\mathrm{a}}$ & 10.85 & $11.98^{\mathrm{a}}$ & $13.10^{\mathrm{a}}$ & $13.60^{\mathrm{a}}$ & 13.16 & 14.51 & 13.87 \\
\hline $\mathrm{CD}=0.05$ & NS & 1.34 & 1.17 & 1.27 & NS & 1.11 & 1.03 & 1.07 & NS & 0.99 & 0.97 & 0.80 & 2.47 & 2.23 & 1.77 \\
\hline $\mathrm{CV} \%$ & & 14.13 & 13.73 & 18.72 & & 9.61 & 10.06 & 12.54 & & 8.93 & 9.64 & 8.7 & 19.42 & 19.47 & 18.27 \\
\hline
\end{tabular}

Means followed by the same letter in a column are not significantly different

*Count/10 leaves

Table 3. Effect of entomopathogenic fungi against Bemisia tabaci

\begin{tabular}{|c|c|c|c|c|c|c|c|c|c|c|c|c|c|c|c|}
\hline \multirow[t]{3}{*}{ Treatments } & \multicolumn{4}{|c|}{ 2014-15 } & \multicolumn{4}{|c|}{$2015-16$} & \multicolumn{4}{|c|}{$2016-17$} & \multirow{2}{*}{\multicolumn{3}{|c|}{$\begin{array}{c}\text { Pooled Mean } \\
\text { Post treatment count }\end{array}$}} \\
\hline & \multirow{2}{*}{$\begin{array}{l}\text { Pre } \\
\text { treat- } \\
\text { ment } \\
\text { count }\end{array}$} & \multicolumn{3}{|c|}{ Post treatment count $*$} & \multirow{2}{*}{$\begin{array}{l}\text { Pre } \\
\text { treat- } \\
\text { ment } \\
\text { count }\end{array}$} & \multicolumn{3}{|c|}{ Post treatment count $*$} & \multirow{2}{*}{$\begin{array}{l}\text { Pre } \\
\text { treat- } \\
\text { ment } \\
\text { count }\end{array}$} & \multicolumn{3}{|c|}{ Post treatment count $*$} & & & \\
\hline & & $\mathrm{I}^{\mathrm{st}}$ spray & $\mathrm{II}^{\text {nd }}$ spray & III $^{\text {rd }}$ spray & & $\mathrm{I}^{\text {st }}$ spray & $\begin{array}{l}\text { II }^{\text {nd }} \\
\text { spray }\end{array}$ & $\begin{array}{l}\mathrm{III}^{\mathrm{rd}} \\
\text { spray }\end{array}$ & & $\mathrm{I}^{\text {st }}$ spray & $\begin{array}{l}\text { II }^{\text {nd }} \\
\text { spray }\end{array}$ & $\begin{array}{l}\mathrm{III}^{\mathrm{rd}} \\
\text { spray }\end{array}$ & $\mathrm{I}^{\mathrm{st}}$ spray & $\begin{array}{l}\mathrm{II}^{\text {nd }} \\
\text { spray }\end{array}$ & $\begin{array}{l}\mathrm{III}^{\text {rd }} \\
\text { spray }\end{array}$ \\
\hline $\begin{array}{l}\text { Metarhizium anisopliae } \\
\text { (AAU-Biometa) }\end{array}$ & 2.19 & $1.16^{\mathrm{c}}$ & $1.12^{\mathrm{c}}$ & $1.10^{\mathrm{b}}$ & 2.28 & $1.17^{\mathrm{cd}}$ & $1.15^{\mathrm{c}}$ & $1.13^{\mathrm{b}}$ & 2.18 & $1.20^{\mathrm{b}}$ & $1.14^{\mathrm{c}}$ & $1.13^{\mathrm{b}}$ & $1.17^{\mathrm{de}}$ & $1.13^{\mathrm{b}}$ & $1.12^{\mathrm{b}}$ \\
\hline $\begin{array}{l}\text { Beauveria bassiana } \\
\text { (AAU-Biosona) }\end{array}$ & 2.18 & $1.10^{\mathrm{c}}$ & $1.12^{\mathrm{c}}$ & $1.05^{\mathrm{b}}$ & 2.30 & $1.13^{\mathrm{cd}}$ & $1.00^{\mathrm{d}}$ & $0.96^{\mathrm{c}}$ & 2.17 & $1.16^{\mathrm{de}}$ & $1.12^{\mathrm{c}}$ & $1.05^{b}$ & $1.13^{\mathrm{de}}$ & $1.08^{b}$ & $0.98^{\mathrm{c}}$ \\
\hline $\begin{array}{l}\text { M. anisopliae } \\
\text { (NBAIRMa-4) }\end{array}$ & 2.20 & $2.05^{\mathrm{b}}$ & $1.11^{\mathrm{c}}$ & $1.13^{\mathrm{b}}$ & 2.34 & $1.98^{\mathrm{b}}$ & $1.16^{\mathrm{c}}$ & $1.17^{\mathrm{b}}$ & 2.25 & $2.00^{\mathrm{c}}$ & $1.20^{\mathrm{c}}$ & $1.13^{b}$ & $1.72^{\mathrm{c}}$ & $1.15^{\mathrm{b}}$ & $1.14^{\mathrm{b}}$ \\
\hline $\begin{array}{l}\text { M. anisopliae } \\
\text { (NBAIRMa-35) }\end{array}$ & 2.18 & $1.98^{\mathrm{c}}$ & $1.20^{\mathrm{c}}$ & $1.11^{\mathrm{b}}$ & 2.50 & $1.69^{\mathrm{b}}$ & $1.32^{\mathrm{b}}$ & $1.06^{\mathrm{bc}}$ & 2.30 & $2.10^{\mathrm{bc}}$ & $1.40^{\mathrm{b}}$ & $1.10^{\mathrm{b}}$ & $1.92^{\mathrm{b}}$ & $1.30^{\mathrm{b}}$ & $1.10^{\mathrm{b}}$ \\
\hline
\end{tabular}




\begin{tabular}{|c|c|c|c|c|c|c|c|c|c|c|c|c|c|c|c|}
\hline $\begin{array}{l}\text { B. bassiana } \\
\text { (NBAIRBb-5a) }\end{array}$ & 2.25 & $1.11^{\mathrm{c}}$ & $1.90^{\mathrm{b}}$ & $0.67^{\mathrm{c}}$ & 2.32 & $1.05^{\mathrm{cd}}$ & $0.98^{\mathrm{d}}$ & $0.63^{\mathrm{d}}$ & 2.28 & $1.12^{\mathrm{e}}$ & $0.87^{\mathrm{d}}$ & $0.64^{d}$ & $1.14^{\mathrm{de}}$ & $0.95^{\mathrm{b}}$ & $0.64^{\mathrm{d}}$ \\
\hline $\begin{array}{l}\text { B. bassiana } \\
\text { (NBAIRBb-23) }\end{array}$ & 2.30 & $1.20^{\mathrm{c}}$ & $1.16^{\mathrm{c}}$ & $1.11^{\mathrm{b}}$ & 2.35 & $1.35^{\mathrm{c}}$ & $1.16^{\mathrm{c}}$ & $1.10^{\mathrm{bc}}$ & 2.20 & $1.30^{\mathrm{d}}$ & $1.18^{\mathrm{c}}$ & $1.10^{\mathrm{b}}$ & $1.28^{\mathrm{d}}$ & $1.16^{\mathrm{b}}$ & $1.10^{\mathrm{b}}$ \\
\hline Imidacloprid $17.8 \mathrm{SL}$ & 2.25 & $1.10^{\mathrm{c}}$ & $0.97^{\mathrm{d}}$ & $0.78^{c}$ & 2.30 & $0.98^{\mathrm{d}}$ & $1.10^{\mathrm{c}}$ & $0.76^{\mathrm{d}}$ & 2.10 & $1.05^{\mathrm{e}}$ & $0.87^{\mathrm{d}}$ & $0.80^{\mathrm{c}}$ & $1.04^{\mathrm{e}}$ & $0.98^{\mathrm{b}}$ & $0.28^{\mathrm{e}}$ \\
\hline Untreated control & 2.30 & $2.32^{\mathrm{a}}$ & $2.40^{\mathrm{a}}$ & $2.25^{\mathrm{a}}$ & 2.25 & $2.45^{\mathrm{a}}$ & $2.38^{\mathrm{a}}$ & $2.30^{\mathrm{a}}$ & 2.45 & $2.40^{\mathrm{a}}$ & $2.32^{\mathrm{a}}$ & $2.27^{\mathrm{a}}$ & $2.33^{\mathrm{a}}$ & $2.25^{\mathrm{a}}$ & $2.10^{\mathrm{a}}$ \\
\hline $\mathrm{CD}=0.05$ & 0.07 & 0.23 & 0.19 & 0.17 & 0.06 & 0.31 & 0.09 & 0.14 & 0.08 & 0.14 & 0.10 & 0.09 & 0.15 & 0.38 & 0.05 \\
\hline $\mathrm{CV} \%$ & 2.14 & 10.45 & 9.43 & 9.81 & 1.71 & 14.36 & 4.59 & 8.14 & 2.56 & 6.32 & 5.40 & 5.14 & 5.80 & 6.49 & 2.40 \\
\hline
\end{tabular}

Means followed by the same letter in a column are not significantly different

*Count/10 leaves

NSKE $2 \%$ recorded $74.37 \%$ mortality to thrips. Arthurs et. al. (2013) observed the maximum efficacy of $B$. bassiana and $M$. anisopliae against $S$. dorsalis compared to other biopesticides. According to Prabhu et. al. (2014), imidacloprid 17.8SL was the best treatments against sucking pests of chillies and was significantly superior to other insecticide molecules.

In respect of yield of Bhut Jalakia, the pooled mean data of three years clearly showed that (Table 4), the highest yield of $52.64 \mathrm{q} / \mathrm{ha}$ was recorded in imidacloprid $17.8 \mathrm{SL}$ treated plot, this was closely followed by NBAIR strain of Bb-5a and $\mathrm{Bb}-23$ with 45.85 and $41.23 \mathrm{q} / \mathrm{ha}$, respectively. The yield of imidacloprid 17.8 SL (52.64 q/ha) was significantly superior to the other plots treated with entomopathogenic fungi. However, no significant difference was observed in between yield parameter of $\mathrm{Bb}-5 \mathrm{a}$ and $\mathrm{Bb}-23$. It could be concluded that all the treatments tested against sucking pests of chilli brought a significant reduction of number of population of sucking pests contributed significantly higher yield as against untreated control.

Table 4. Effect of local and NBAIR strains on yield of Bhut Jalakia

\begin{tabular}{|c|c|c|c|c|}
\hline Treatments & $\begin{array}{l}2014-15 \\
(\mathrm{q} / \mathrm{ha})\end{array}$ & $\begin{array}{l}\text { 2015-16 } \\
\text { (q/ha) }\end{array}$ & $\begin{array}{l}2016-17 \\
(\mathrm{q} / \mathrm{ha})\end{array}$ & $\begin{array}{l}\text { Pooled } \\
\text { mean }\end{array}$ \\
\hline $\begin{array}{l}\text { Metarhizium anisopliae } \\
\text { (AAU strain) - Biometa }\end{array}$ & $28.75^{\mathrm{d}}$ & $31.00^{\mathrm{de}}$ & $31.88^{\mathrm{e}}$ & 30.54 \\
\hline $\begin{array}{l}\text { Beauveria bassiana } \\
\text { (AAU strain) - Biosona }\end{array}$ & $40.20^{c}$ & $34.63^{\mathrm{cd}}$ & $32.48^{\mathrm{e}}$ & 35.77 \\
\hline $\begin{array}{l}\text { M. anisopliae (Ma-4) } \\
\text { NBAIR strain }\end{array}$ & $29.75^{\mathrm{d}}$ & $29.50^{\mathrm{e}}$ & $34.70^{\mathrm{d}}$ & 31.32 \\
\hline $\begin{array}{l}\text { M. anisopliae (Ma-35) } \\
\text { NBAIR strain }\end{array}$ & $30.38^{\mathrm{d}}$ & $30.38^{\mathrm{e}}$ & $38.20^{\mathrm{c}}$ & 32.99 \\
\hline $\begin{array}{l}\text { B. bassiana }(\mathrm{Bb}-5 \mathrm{a}) \mathrm{NBAIR} \\
\text { strain }\end{array}$ & $51.20^{\mathrm{ab}}$ & $42.00^{\mathrm{b}}$ & $44.35^{b}$ & 45.85 \\
\hline $\begin{array}{l}\text { B. bassiana }(\mathrm{Bb}-23) \text { NBAIR } \\
\text { strain }\end{array}$ & $46.00^{\mathrm{b}}$ & $37.88^{\mathrm{c}}$ & $39.80^{\mathrm{c}}$ & 41.23 \\
\hline Imidacloprid $17.8 \mathrm{SL}$ & $53.83^{\mathrm{a}}$ & $50.75^{\mathrm{a}}$ & $53.33^{\mathrm{a}}$ & 52.64 \\
\hline Untreated control & $26.75^{\mathrm{d}}$ & $24.13^{\mathrm{f}}$ & $29.45^{\mathrm{f}}$ & 26.78 \\
\hline $\mathrm{CD}=0.05$ & 5.92 & 3.78 & 1.83 & 5.59 \\
\hline $\mathrm{CV} \%$ & 10.49 & 7.34 & 4.62 & 8.60 \\
\hline
\end{tabular}

Means followed by the same letter in a column are not significantly different
All the entomopathogenic fungi evaluated during the course of study exhibited equally more or less efficacies in suppressing the population of sucking pests of Bhut Jalakia compared to untreated control.

\section{ACKNOWEDGEMENT}

The authors are grateful to the Director, NBAIR, Bengaluru, Karnataka, India, for providing necessary entomopathogenic fungi to conduct the experiment. The authors also acknowledge the support received from Director of Research (Agri.) and Professor and Head, Department of Entomology, Assam Agricultural University, Jorhat, Assam, India.

\section{REFFERENCES}

Arthurs SP, Aristizábal LF, Avery PB. 2013. Evaluation of entomopathogenic fungi against chillithrips, Scirtothrips dorsalis. J Insect Sci. 13: 1536-2442. https://doi.org/10.1673/031.013.3101 PMid:23895429 PMCid:PMC3735053

Baruah S, Zaman Md. K, Rajbongshi P, Das S. 2014. A Review on Recent researches on Bhut Jalakia and where the pharmacological activity of capsaicin. Intl J Pharm Sci Rev Res. 24: 89-94.

Begam N, Saikia DK, Borkakati RN. 2016. Seasonal incidence of major insect-pests and their natural enemies of Bhut Jalakia. Ann Pl Protec Sci. 24: 259-264.

Berke T, Sheih SC. 2000. Chilli peppers in Asia. Capsicum Egg Pl Newslett. 19: 38-41.

Bhagowati RR, Chankija S. 2009. Genetic variability and traditional practices in Naga King chilli landraces of Nagaland. Asian Agri History 3: 171-180.

Buragohain P, Saikia DK, Borkakati RN, Dutta BC, Thangjam R. 2017. Pest complex and the population dynamics of major pests of Bhut Jalakia. Ecol Environ Conser. 23: 265-272.

Das G. 2013. Efficacy of imidacloprid 17.8SL, a nicotinoid group of insecticide against the infestation of chilli 
aphid, Myzus persicae (Hemiptera: Aphididae). Int $J$ Biol Sci. 2: 154-159.

Eidy M, Rafiee-Dastjerdi H, Zargarzadeh F, Golizadeh A and Mahdavi V. 2016. Pathogenicity of the entomopathogenic fungi Beauveria bassiana (Balsamo) and Verticillium lecanii (Zimmerman) against aphid Macrosiphum rosae Linnaeus (Hemiptera: Aphididae) under laboratory conditions. Jordan J Biol Sci. 9: 25-28. https://doi.org/10.12816/0027004

Jagdish EJ, Purnima AP. 2011. Evaluation of selective botanicals and entomopathogens against Scirtothrips dorsalis Hood under poly house conditions on rose. $J$ Biopesticides 4: 81-85, 244.

Jandricic SE, Filotas M, Sanderson JP, Wraight SP. 2014. Pathogenicity of conidia-based preparations of entomopathogenic fungi against the greenhouse pest aphids Myzus persicae, Aphis gossypii, and Aulacorthum solani (Hemiptera: Aphididae). J Invertebr Pathol. 118: 34-46. https://doi.org/10.1016/j.jip.2014.02.003 PMid:24583227

Kim JJ, Jeong G, Han JH, Lee S. 2013. Biological control of aphid using fungal culture and culture filtrates of Beauveria bassiana. Mycobiology 41: 221-224. https://doi.org/10.5941/MYCO.2013.41.4.221 PMid:24493943 PMCid:PMC3905126
Lee WW, Shin TY, Bae SM, Woo SD. 2015. Screening and evaluation of entomopathogenic fungi against the green peach aphid, Myzus persicae using multiple tools. J Asia-Pacific Entomol. 18: 607-615. https://doi.org/10.1016/j.aspen.2015.07.012

Loureiro ES, Moino JRA. 2006. Pathogenicity of hyphomycetes fungi to aphids Aphis gossypii Glover and Myzus persicae (Sulzer) (Hemiptera: Aphididae). Neotrop Entomol. 35: 660-665. https://doi.org/10.1590/S1519-566X2006000500014

Mahalingappa PB, Reddy KD, Reddy KN, Subbaratnam GV. 2006. Dissipation of ethion and chlorpyriphos residues in/ on chilli (Capsicum annuum). Pesticide Res J. 18: 72-73.

PatilAS, Patil PD, Patil RS. 2002. Efficacy of different schedule doses of imidacloprid against sucking pest complex of chilli (Capsicum annum L.). Pestology 26: 31-33.

Prabhu ST, Nagraja MV, Ganapathi T. 2014. Evaluation of bio-efficacy of imidacloprid $17.8 \% \mathrm{~S}$ L against chilli insect pests. Trends Biosci. 7: 3331-3334.

Sanatombi K, Sharma GJ. 2008 In vitro plant regeneration in six cultivars of Capsicum spp. using different explants. Biol Plant. 52: 141-145. https://doi.org/10.1007/s10535-008-0029-0 